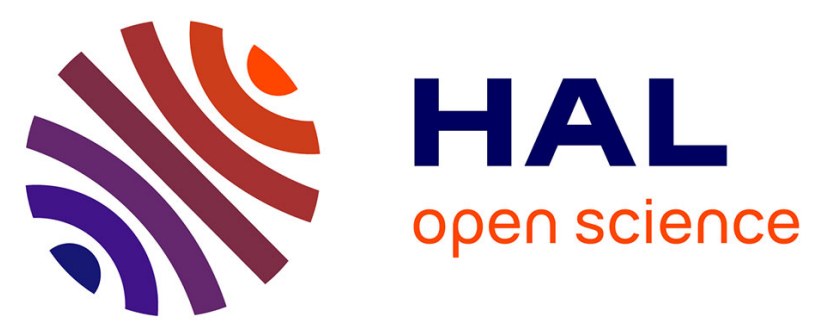

\title{
Impact of dispersion forces on the atomic structure of a prototypical network-forming disordered system: The case of liquid GeSe2
}

Évelyne Martin, Assil Bouzid, Guido Ori, Mauro Boero, Carlo Massobrio

\section{- To cite this version:}

Évelyne Martin, Assil Bouzid, Guido Ori, Mauro Boero, Carlo Massobrio. Impact of dispersion forces on the atomic structure of a prototypical network-forming disordered system: The case of liquid GeSe2. Journal of Chemical Physics, 2017, 147 (4), pp.044504. 10.1063/1.4986166 . hal-02349387

\section{HAL Id: hal-02349387 \\ https://hal.science/hal-02349387}

Submitted on 18 Jan 2022

HAL is a multi-disciplinary open access archive for the deposit and dissemination of scientific research documents, whether they are published or not. The documents may come from teaching and research institutions in France or abroad, or from public or private research centers.
L'archive ouverte pluridisciplinaire HAL, est destinée au dépôt et à la diffusion de documents scientifiques de niveau recherche, publiés ou non, émanant des établissements d'enseignement et de recherche français ou étrangers, des laboratoires publics ou privés. 


\section{Impact of dispersion forces on the atomic structure of a prototypical network-forming}

disordered system: the case of liquid $\mathrm{GeSe}_{2}$

Evelyne Lampin, ${ }^{1}$ Assil Bouzid, ${ }^{2}$ Guido Ori, ${ }^{3}$ Mauro Boero, ${ }^{3}$ and Carlo Massobrio ${ }^{3}$

1)Univ. Lille, CNRS, Centrale Lille, ISEN, Univ. Valenciennes, UMR 8520 - IEMN, F-59000 Lille, France

${ }^{2)}$ Chaire de Simulation à l'Echelle Atomique (CSEA), Ecole

Polytechnique Fédérale de Lausanne (EPFL), CH-1015 Lausanne,

\section{Switzerland}

3) Université de Strasbourg, CNRS, Institut de Physique et Chimie

des Matériaux de Strasbourg, UMR 7504, F-67034 Strasbourg,

\section{France}

(Dated: January 18, 2022)

A set of structural properties of liquid $\mathrm{GeSe}_{2}$ are calculated by using first-principles molecular dynamics and including, for the first time, van der Waals dispersion forces. None of the numerous atomic-scale simulations performed in the past on this prototypical disordered network-forming material had ever accounted for dispersion forces in the expression of the total energy. To this purpose, we employed either the Grimme-D2 or the maximally localized Wannier functions scheme. We assessed the impact of the dispersion forces on properties such as the partial structure factors, the pair correlation functions, the bond angle distribution and the number of corner vs edge sharing connections. The maximally localized Wannier functions scheme is more reliable than the Grimme-D2 scheme in reproducing existing first-principles results. In particular, the Grimme-D2 scheme worsens the agreement with the experiments in the case of the Ge-Ge pair correlation function. Our study shows that the impact of dispersion forces on disordered chalcogenides has to be considered with great care, since it cannot be necessarily the same when adopting different recipes.

PACS numbers: 61.25.Em, 61.20.Ja, 71.15.Pd 


\section{INTRODUCTION}

The proper account of dispersive (van der Waals, vdW) contributions is a challenging task for atomic-scale computational material science. In this context, $\mathrm{A}_{x} \mathrm{~B}_{1-x}(\mathrm{~A}=\mathrm{Ge}, \mathrm{Si} ; \mathrm{B}=\mathrm{O}, \mathrm{Se}, \mathrm{S})$ disordered network-forming materials are worth investigating, since made of atomic species qualitatively prone to be denoted as cations and anions and yet bearing close electronegativities. Such combination of physical effects results in cohesive forces of iono-covalent nature. The question arises on whether there might be, as a function of the composition $x$ and for given families of compounds, bonding situations for which dispersion forces cannot be merely neglected by invoking efficient screening of other predominant bonding contributions.

Based on a well-established series of achievements, it appears that first-principles molecular dynamics (FPMD) in the framework of density functional theory (DFT) is a reliable tool to gain insight into the properties of glasses and liquids such as $\mathrm{Ge}_{x} \mathrm{Se}_{1-x}, \mathrm{Ge}_{x} \mathrm{Te}_{1-x}$, or their ternary counterparts $^{1-16}$. For these systems, the number of results obtained without an explicit account of dispersion forces largely exceeds those for which such forces have been explicitly considered. However, it is nowadays widely accepted that the mere use of the available exchange-correlation (XC) functionals cannot systematically ensure a proper account of long-range electron correlations responsible for van der Waals contributions. For instance, this was shown to be the case for liquid $\mathrm{Ge}_{15} \mathrm{Te}_{85}$, ${ }^{17}$ pointing out the need of additional studies extended to other telluride and/or chalcogenide disordered materials.

Along these lines, and focusing on glassy $\mathrm{GeTe}_{4}$, the impact of dispersion forces and its interplay with the choice of the XC functionals within DFT was carefully analyzed. In Ref. 18, we compared the levels of agreement on structural properties between experiments and theory (FPMD), as predicted by a set of different modelling schemes. Four different theoretical recipes were considered, each one corresponding to a distinct structural model. These were obtained by selecting either the PBE (Perdew, Burke and Ernzerhof) ${ }^{19}$ or the BLYP (Becke, Lee, Yang and Parr $)^{20,21}$ XC functionals and, for each one of the two, by disregarding or including van der Waals dispersion forces via the Grimme contribution. ${ }^{22,23}$ The inclusion of the vdW forces lead to better performances and realistic structural predictions in the case of both the PBE and the BLYP exchange-correlation functionals.

In view of this result, the main motivation of the present work is to assess further the impact of dispersion forces on a representative case of disordered chalcogenide, liquid $\mathrm{GeSe}_{2}$. The presence 
of a predominant structural unit (the $\mathrm{GeSe}_{4}$ tetrahedron) coexisting with homopolar bonds makes the atomic structure of liquid $\mathrm{GeSe}_{2}$ challenging to elucidate for both experiments and atomicscale modelling. ${ }^{24-31}$ As shown by FPMD, the description of both short and intermediate range order of liquid $\mathrm{GeSe}_{2}$ depends on the choice of a specific exchange-correlation functional. ${ }^{32,33}$ In particular, generalized gradient approximated (GGA) functionals favoring electronic localization bring calculations in better agreement with structural data. Substantial improvements were found when introducing the Perdew-Wang (PW) recipe at the place of the local density approximation (LDA) and, in a further step, the BLYP scheme at the place of the PW scheme. ${ }^{20,21,32-35}$ In the case of liquid $\mathrm{GeSe}_{2}$, the partial structure factors obtained via DFT-FPMD were able to reproduce experimental data for an extended range of wavevectors in reciprocal space, the same consideration holding for pair correlation functions in real space. ${ }^{27,33}$ However, a number of differences between theory and experiments did persist, as the underestimate of the first sharp diffraction peak (FSDP) in the Ge-Ge partial structure factor. Such shortcoming is responsible for the absence of the FSDP in the Bhatia-Thornton concentration-concentration structure factor ${ }^{36}$ and it was found to be largely independent on the periodic cell dimensions, as shown by taking a number of atoms $N$ $=480$ instead of $N=120 .{ }^{37}$ Since the occurrence of such FSDP could not be ascribed to trivial system size effects, the consideration of other plausible origins for the residual lack of agreement with experiments is very much in order. Therefore, one of our main motivation is to focus on the dispersion forces as a missing ingredient in the theoretical description of this chalcogenide. ${ }^{36}$ Our approach complements available indications on the impact of dispersion forces on $\mathrm{Se} / \mathrm{S}$ made disordered chalcogenides, as those rationalized in Ref. 38.

In the present work, we produce a new set of FPMD data on liquid $\mathrm{GeSe}_{2}$ by including dispersion forces in the FPMD Kohn-Sham hamiltonian. Two different methodologies are employed. One consists in following the formalism due to Grimme and, specifically, the D2 formula. ${ }^{22} \mathrm{We}$ also employed a scheme more profoundly rooted into the density functional theory in the sense that the electronic structure, obtained within the selected DFT-GGA framework, is used to compute the maximally localized Wannier functions (MLWF) on which the present van der Waals scheme is based. ${ }^{39-41}$

Our results show that the account of dispersion forces does not alter significantly the structural properties of liquid $\mathrm{GeSe}_{2}$. However, the Grimme scheme is unable to reproduce correctly the experimental profile of the Ge-Ge pair correlation function, resulting in a perceptible underestimate of the number of corner-sharing connections. The level of agreement is restored when using 
the MLWF formalism, confirming its superior performances in terms of correct reproduction of physical properties closely related to chemical bonding. We found no impact of vdW forces on the concentration-concentration structure factor over the full range of wavevectors in reciprocal space.

This work is organized as follows. Section II provides details on our theoretical model, that is based on the first-principles molecular dynamics framework. Section III contains structural information extracted from the partial structure factors in the Faber-Ziman and Bhatia-Thornton formalisms. Section IV is devoted to the partial pair correlation functions and to a description of the network topology via the counting of the edge-sharing and corner-sharing connections. Conclusive remarks are collected in Section V.

\section{THEORETICAL MODEL}

The theoretical framework of our calculations is identical to the one employed and detailed extensively in previous papers devoted to liquid $\mathrm{GeSe}_{2} \cdot{ }^{27,33}$ In the present case, FPMD simulations have been performed at constant volume on a system containing 240 (80 Ge and $160 \mathrm{Se}$ ) atoms positioned in a periodically repeated cubic cell of size $19.76 \AA$, this value allowing to recover the experimental density of the liquid ${ }^{42}$ at the desired temperature $(T=1050 \mathrm{~K}) . N=240$ turns out to be a suitable system size to obtain reliable structural properties of liquid $\mathrm{GeSe}_{2}$ at both short and intermediate ranges. This choice is substantiated by our comparative work on system sizes $N$ $=120$ and $N=480$, clearly demonstrating that all results obtained in the past with $N=120$ were not significantly altered when moving to $N=480 .{ }^{37}$ We recall that the electronic structure was described within density functional theory and evolved self-consistently in time by Car-Parrinello molecular dynamics (CPMD) $)^{43}$ and the cpmd code. ${ }^{44}$ The BLYP generalized gradient approximation was used, based on the exchange energy obtained by Becke ${ }^{20}$, and the correlation energy according to Lee, Yang and Parr. ${ }^{21}$ Valence electrons have been treated explicitly, in conjunction with norm conserving pseudopotentials of the Trouiller-Martins type to account for core-valence interactions. ${ }^{45}$ The wave functions have been expanded at the $\Gamma$ point of the supercell on a plane wave basis set with an energy cutoff $E_{c}=20 \mathrm{Ry}$. Long-range dispersion forces were accounted for in two distinct manners. The first consists in using the DFT-D2 formula proposed by Grimme ${ }^{22}$ and it will be termed vdW1 in what follows. We stress the fact that, although empirical, such a vdW correction is a thorough DFT-based formulation in which the parameters are self-consistently 
tuned on different functionals, including the one used in this work, and benchmarked on a wealth of different systems from simple molecules to complex reactive surfaces and chalcogenides. ${ }^{46-48}$ No experimental parameters are considered in the construction of this specific vdW correction and its inclusion does not affect at any stage the Kohn-Sham equations, ${ }^{49,50}$ thus preserving the first-principle character of all electronic structure calculations. However, the Grimme formalism does not allow for any update of the vdW interactions as a result of the changes in the electronic structure that occur during the dynamical evolution. To go beyond this limitation, we employed a second $\mathrm{vdW}$ scheme in which the vdW coefficients are recalculated for each ion of the system, becoming explicitly time-dependent. ${ }^{39-41}$ This scheme (referred to as vdW2 in what follows) makes use of the electronic wavefunctions via the unitary transformation of the Kohn-Sham orbitals giving the maximally localized Wannier functions. Such strategy has rapidly acquired an extended record of reliability for several test cases. ${ }^{41,51,52}$

In the FPMD approach, a fictitious electron mass of 2000 a.u. (i.e. in units of $m_{e} a_{0}^{2}$ where $m_{e}$ is the electron mass and $a_{0}$ is the Bohr radius), and a time step of $\Delta t=0.12 \mathrm{fs}$ have been used to integrate the equations of motion. The control of the temperature has been implemented for both the ionic and electronic degrees of freedom by using Nosé-Hoover thermostats. ${ }^{53-55}$ The initial coordinates have been obtained by selecting from a periodic cell of $N=480$ atoms (previously employed to produce the results of Ref. 37) the positions of the inner 240 atoms. As a first step, the system has been brought to $T=0 \mathrm{~K}$ by structural optimization via forces minimization, so as to avoid any artifact due to the presence of spurious interactions that might be due to our initial system construction. FPMD runs lasting in between 30 ps and 100 ps have been produced at increasing temperatures without accounting for vdW contributions to bring the system at the production temperature of $T=1050 \mathrm{~K}$ in the liquid phase. After collecting statistical averages over a period of $50 \mathrm{ps}$, the final configuration is stored to ensure the same initial starting point for simulations runs involving the vdW1 and vdW2 dispersion forces. In both cases, the liquid is first cooled at $T=300 \mathrm{~K}$ and then heated up over a time interval of $115 \mathrm{ps}$ for vdW 1 and 170 ps for vdW2. Statistical averages pertaining to the vdW1 and vdW2 situations were collected over $30 \mathrm{ps}$ and $42 \mathrm{ps}$ respectively. The different length of time for the collection of the statistical averages has no special significance, both trajectories being sufficiently extended to access thermal equilibrium. The statistical errors for the models employed have been found to be very similar. We make inferences on the impact of the vdW1 and the vdW2 schemes for a set of properties in reciprocal and direct space. Our references for comparison are the experimental data of Ref. 24 
obtained from isotopic substitution in neutron diffraction and the present FPMD results with no consideration of dispersion forces (NvdW hereafter). For all sets of calculations, unless otherwise stated, statistical errors are limited to $5 \%$ at most.

\section{STRUCTURE FACTORS}

\section{A. Partial structure factors: Faber-Ziman and Bhatia-Thornton results}

The calculated Faber-Ziman (FZ) partial structure factors for liquid $\mathrm{GeSe}_{2}$ are shown in Fig. 1 . The FPMD sets of results are very close to each other in the case of the partial structure factors $S_{\mathrm{GeSe}}^{\mathrm{FZ}}(k)$ and $S_{\mathrm{SeSe}}^{\mathrm{FZ}}(k)$. However, the intensity of the main peak is significantly higher in the vdW1 case for $S_{\mathrm{SeSe}}^{\mathrm{FZ}}(k)$. By focusing on the FSDP region around $k \sim 1 \AA^{-1}$, there is a vanishing impact of the vdW corrections on the intermediate range properties associated with Ge-Se correlations. In the case of $S_{\mathrm{GeGe}}^{\mathrm{FZ}}(k)$, there are some discernible variations in the peak intensities at $k \sim 1 \AA^{-1}$. Such differences are of little relevance in the context of the present investigation since mostly related to statistical errors comparable to those found when moving from $N=120$ to $N=480$. Worth of notice for $S_{\mathrm{GeGe}}^{\mathrm{FZ}}(k)$ are the intensities of the maximum and of the minimum located at $k \sim 2 \AA^{-1}$ and $k \sim 3 \AA^{-1}$, respectively. These are more pronounced in the case of vdW1 when compared to the vdW2 and NvdW models, the three values lying within the experimental statistical errors. Taken together, it remains true that the analyses of the partial structure factors in the FZ formalism do not bring any striking evidence on the possible occurrence of structural changes due to dispersion forces. We recorded some minor effects in $S_{\mathrm{SeSe}}^{\mathrm{FZ}}(k)$ and $S_{\mathrm{GeGe}}^{\mathrm{FZ}}(k)$.

It is of interest to seek further evidence on the impact of dispersion forces by considering the Bhatia-Thornton partial structure factors ${ }^{56} S_{\mathrm{NN}}(k)$ (number-number), $S_{\mathrm{NC}}(k)$ (numberconcentration) and $S_{\mathrm{CC}}(k)$ (concentration-concentration) (Fig. 2).

These can be obtained by linear combinations of the Faber-Ziman partial structure factors as follows:

$$
\begin{array}{r}
S_{\mathrm{NN}}(k)=c_{\mathrm{Ge}} c_{\mathrm{Ge}} S_{\mathrm{GeGe}}^{\mathrm{FZ}}(k)+c_{\mathrm{Se}} c_{\mathrm{Se}} S_{\mathrm{SeSe}}^{\mathrm{FZ}}(k)+ \\
2 c_{\mathrm{Ge}} c_{\mathrm{Se}} S_{\mathrm{GeSe}}^{\mathrm{FZ}}(k), \\
S_{\mathrm{NC}}(k)=c_{\mathrm{Ge}} c_{\mathrm{Se}}\left[c_{\mathrm{Ge}}\left(S_{\mathrm{GeGe}}^{\mathrm{FZ}}(k)-S_{\mathrm{GeSe}}^{\mathrm{FZ}}(k)\right)\right. \\
\left.-c_{\mathrm{Se}}\left(S_{\mathrm{SeSe}}^{\mathrm{FZ}}(k)-S_{\mathrm{GeSe}}^{\mathrm{FZ}}(k)\right)\right],
\end{array}
$$




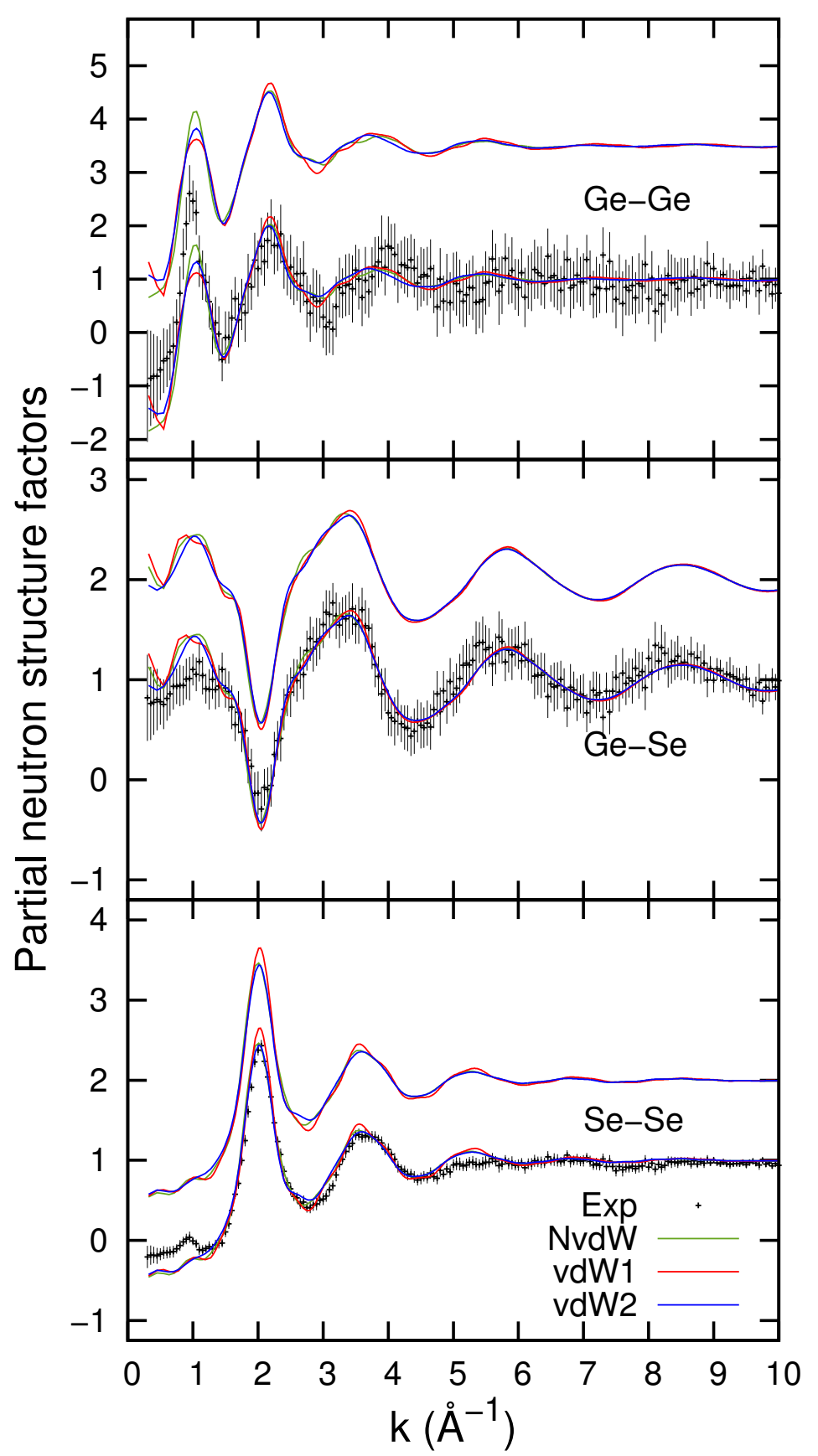

Figure 1. (Color online) The Faber-Ziman partial structure factors $S_{\mathrm{GeGe}}^{\mathrm{FZ}}(k)$ (top panel), $S_{\mathrm{GeSe}}^{\mathrm{FZ}}(k)$ (middle panel) and $S_{\mathrm{SeSe}}^{\mathrm{FZ}}(k)$ (bottom panel) for liquid $\mathrm{GeSe}_{2}$ at $T=1050 \mathrm{~K}$. Experimental data from Ref. 24 (crosses with error bars) are compared to results for the models NvdW (green line), vdW1 (red line) and vdW2 (blue line). The results of calculations are shown by including (lower curve) and by not including (higher curve) the experimental results in the comparison. 


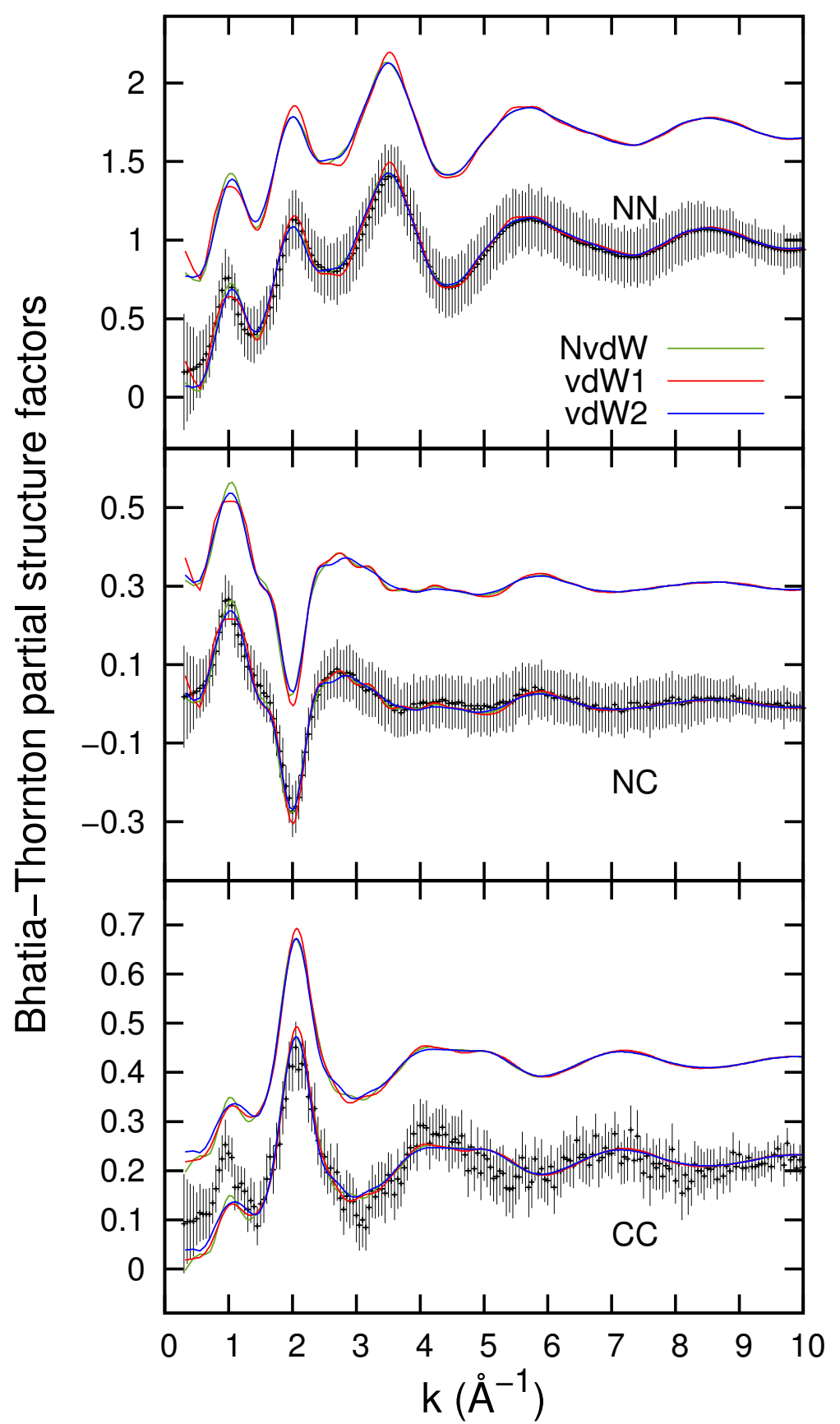

Figure 2. (Color online) The Bhatia-Thornton partial structure factors $S_{\mathrm{NN}}(k)$ (top panel), $S_{\mathrm{NC}}(k)$, (middle panel) and $S_{\mathrm{CC}}(k)$ (bottom panel) for liquid $\mathrm{GeSe}_{2}$ at $T=1050 \mathrm{~K}$. Experimental data from Ref. 24 (crosses with error bars) are compared to results for the models NvdW (green line), vdW1 (red line) and vdW2 (blue line). The results of calculations are shown by including (lower curve) and by not including (higher curve) the experimental results in the comparison. 


$$
\begin{aligned}
S_{\mathrm{CC}}(k)= & c_{\mathrm{Ge}} c_{\mathrm{Se}}\left\{1+c_{\mathrm{Ge}} c_{\mathrm{Se}}\left[\left(S_{\mathrm{GeGe}}^{\mathrm{FZ}}(k)-S_{\mathrm{GeSe}}^{\mathrm{FZ}}(k)\right)\right.\right. \\
& \left.\left.+\left(S_{\mathrm{SeSe}}^{\mathrm{FZ}}(k)-S_{\mathrm{GeSe}}^{\mathrm{FZ}}(k)\right)\right]\right\} .
\end{aligned}
$$

where $c_{\mathrm{Ge}}$ and $c_{\mathrm{Se}}$ denote respectively the atomic fraction of $\mathrm{Ge}$ and $\mathrm{Se}$.

We recall that due to very close values of the coherent scattering length of the chemical species Ge and Se $\left(b_{G e}=8.185 \mathrm{fm}, b_{S e}=7.97 \mathrm{fm}\right)^{57}$ and to the limited range of variation of $S_{\mathrm{NC}}(k)$ and $S_{\mathrm{CC}}(k), S_{\mathrm{NN}}(k)$ is a very good approximation of the total structure factor, i.e. $\left|S_{\mathrm{T}}(k)-S_{\mathrm{NN}}(k)\right|<0.025$. Therefore, the considerations developed hereafter on $S_{\mathrm{NN}}(k)$ apply equally well to the total neutron structure factor $S_{T}(k)$.

There are no discernible effects of the dispersion forces on the Bhatia-Thornton partial structure factors $S_{\mathrm{NC}}(k)$ and $S_{\mathrm{CC}}(k)$. In particular, one notice the persistent absence (or the large underestimate) of the FSDP in all calculated $S_{\mathrm{CC}}(k)$ strongly contrasting with the prominent feature found in neutron diffraction experiments. ${ }^{24}$ Given these results, one can safely rule out any impact of $\mathrm{vdW}$ forces on the intensity of the FSDP, thereby adding another piece of information toward precise understanding of this longstanding open issue. The Bhatia-Thornton partial structure factors $S_{\mathrm{NN}}(k)$ features higher peak intensities at $k \sim 2 \AA^{-1}$ and $k \sim 3.5 \AA^{-1}$ when adopting the vdW1 recipe. Although these variations are minimal and within the statistical error of the experimental measurements, this is in line with the previous observation of the $S_{\mathrm{GeGe}}^{\mathrm{FZ}}(k)$ and $S_{\mathrm{SeSe}}^{\mathrm{FZ}}(k)$ cases, indicating that the Grimme approach deviates more from the experimental pattern than the MLWF scheme.

\section{REAL SPACE PROPERTIES}

\section{A. Partial pair-correlation functions}

Fig. 3 provides a comparison between the three sets of calculations NvdW, vdW1 and vdW2 of the pair correlation functions $g_{\mathrm{GeGe}}(r), g_{\mathrm{GeSe}}(r)$ and $g_{\mathrm{SeSe}}(r)$, and the corresponding experimental results. Integration of the pair-correlation function $g_{\mathrm{GeSe}}(r)$ over the first shell of neighbors leads to coordination numbers $\bar{n}_{\mathrm{GeSe}}$ lying within $6 \%\left(\bar{n}_{\mathrm{GeSe}}(\exp )=3.5, \bar{n}_{\mathrm{GeSe}}(\mathrm{NvdW})=3.7, \bar{n}_{\mathrm{GeSe}}(\mathrm{vdW} 1)\right.$ $\left.=3.7, \bar{n}_{\mathrm{GeSe}}(\mathrm{vdW} 2)=3.6\right)$. These numbers exemplify the lack of sensitivity of Ge-Se correlations to the presence of dispersion forces, in agreement with the predominant ionic contribution that can be assumed to be responsible of most of the Ge-Se bonding.

In the case of $g_{\mathrm{SeSe}}(r)$ the experimental value for $\bar{n}_{\mathrm{SeSe}}$ is $\bar{n}_{\mathrm{SeSe}}(\exp )=0.23$. With our calcula- 
tions we obtain $\bar{n}_{\mathrm{SeSe}}(\mathrm{NvdW})=0.20, \bar{n}_{\mathrm{SeSe}}(\mathrm{vdW} 1)=0.19, \bar{n}_{\mathrm{SeSe}}(\mathrm{vdW} 2)=0.25$. Consideration of these numbers and visual inspection of Fig. 3 are indicative of a broad level of agreement among the three sets of data. However, the Grimme recipe (vdW1) stands out as the farthest apart from the experimental prediction when focusing on the second main peak at about $4 \AA$.

The case of $g_{\mathrm{GeGe}}(r)$ is worth dwelling on in deeper detail. This partial pair correlation function has been recognized as the one featuring the least satisfactory agreement with experiments, despite an overall quite acceptable behavior in terms of coordination numbers due to the use of the BLYP exchange correlation functional. ${ }^{33}$ The partial pair correlation function $g_{\mathrm{GeGe}}(r)$ features a three peak structure for $r<4 \AA$, representative of homopolar bonds, edge-sharing (ES) and corner-sharing (CS) connections. The pattern corresponding to these marks is reproduced by all FPMD models, with unequal intensities of the first peak corresponding to homopolar bonds. These differences are partially compensated by a broad agreement on the values of the coordination numbers $\bar{n}_{\mathrm{GeGe}}(\mathrm{NvdW})=0.18, \bar{n}_{\mathrm{GeGe}}(\mathrm{vdW} 1)=0.19, \bar{n}_{\mathrm{GeGe}}(\mathrm{vdW} 2)=0.23$ all of them lying close to the experimental result, 0.25 . We recall that sizeable variations on the intensity and width of the first peak are somewhat expected in view of the extended temporal persistence of Ge-Ge homopolar bonds that might form on specific trajectories. For the purpose of the present work, it is of interest instead to focus on the heights of the second and of the third peak of $g_{\mathrm{GeGe}}(r)$. The relative intensities of these peaks is clearly different in the vdW1 case, with a third peak standing out well beyond any expected statistical error. This corresponds to an overestimate of corner-sharing connections induced by the Grimme approximation. As a valuable alternative, MLWF succeed in leaving unaltered the quite acceptable profile of the calculated $g_{\mathrm{GeGe}}(r)$, thereby providing a first, clear-cut evidence of its better performances for this kind of network structures.

\section{B. Bond angle distributions and network topology}

In Fig. 4 , we show the Se-Ge-Se $\left(\theta_{\mathrm{SeGeSe}}\right)$ and Ge-Se-Ge $\left(\theta_{\mathrm{GeSeGe}}\right)$ bond angle distributions. The shapes of $\theta_{\text {SeGeSe }}$ for the three cases are nearly identical and highly symmetrical, pointing out a predominant tetrahedral arrangement, with angles distributed around the expected value $109^{\circ}$. In this respect, the dispersion forces do not alter the regularity of the tetrahedral structures. Turning to $\theta_{\mathrm{GeSeGe}}$, the two distinct peaks visible at about $80^{\circ}$ and $100^{\circ}$ can be assigned to the formation of edge- and corner-sharing tetrahedral connections, respectively. ${ }^{27,28,33}$ Changes in the edge-sharing/corner-sharing peak intensities ratio suggest that corner-sharing connections are 


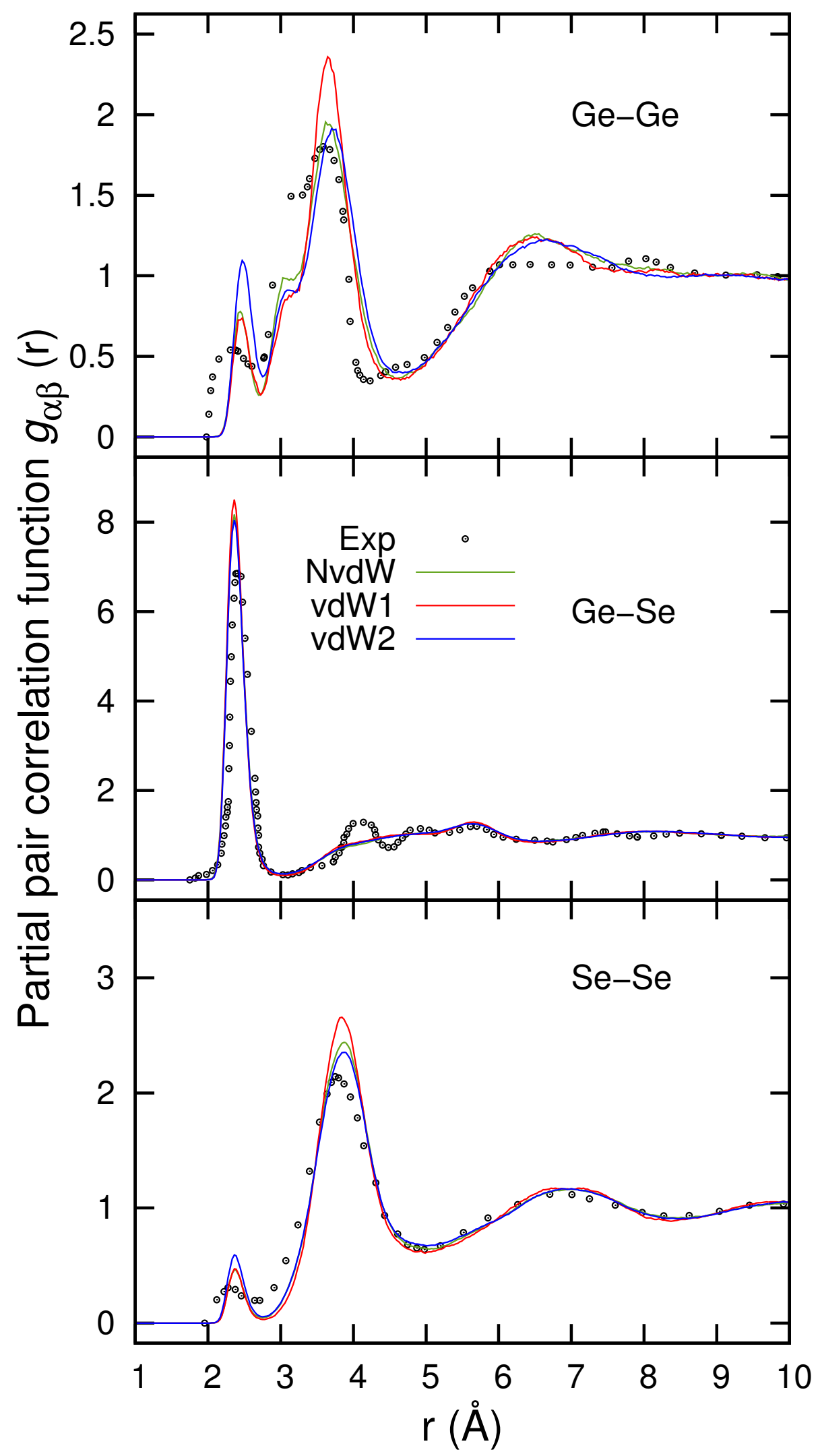

Figure 3. (Color online) The partial pair correlation functions $g_{\mathrm{GeGe}}(r)$ (top panel), $g_{\mathrm{GeSe}}(r)$ (middle panel) and $g_{\mathrm{SeSe}}(r)$ (bottom panel) for liquid $\mathrm{GeSe}_{2}$ at $T=1050 \mathrm{~K}$. Open small circles are the experimental data 


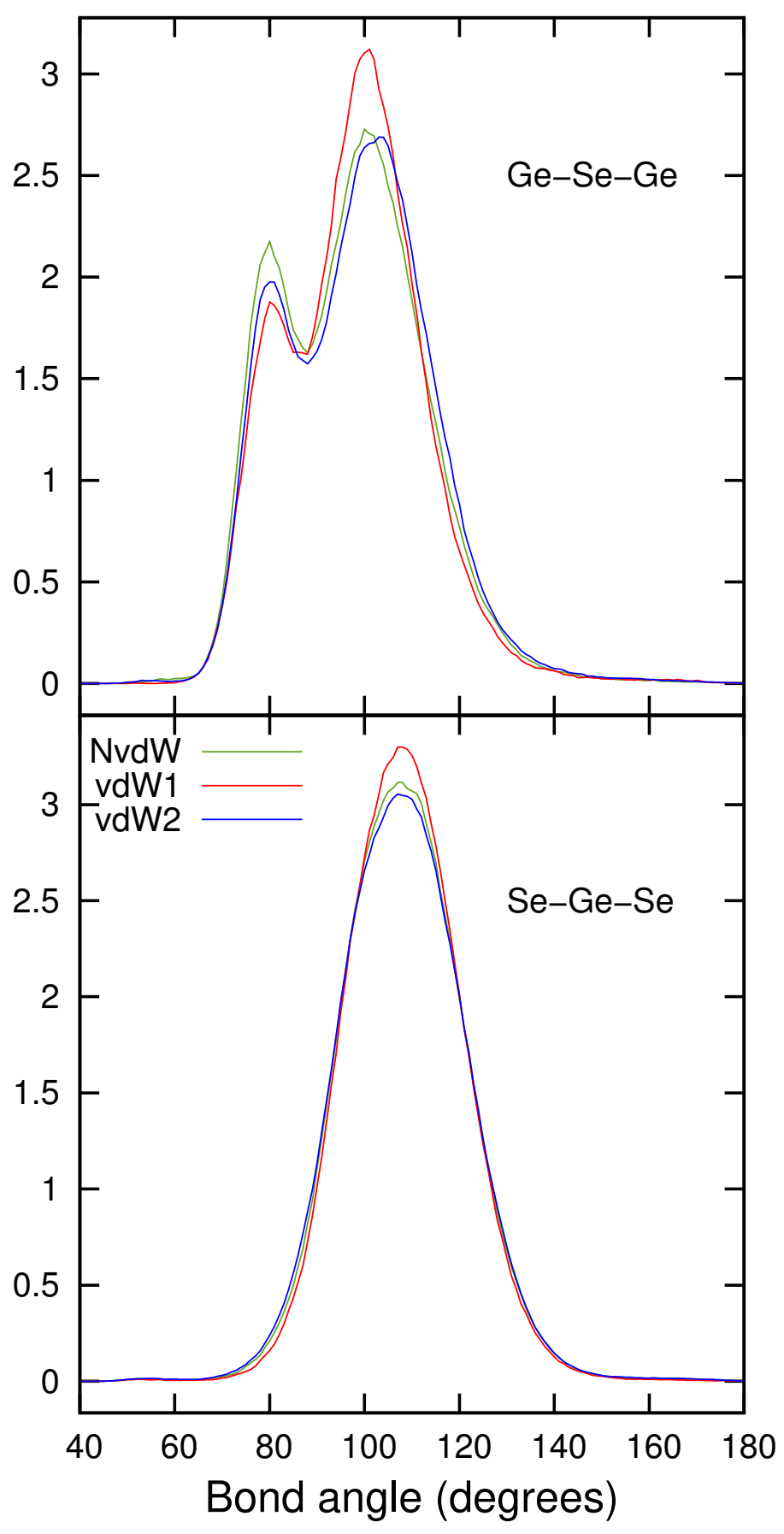

Figure 4. (Color online) The calculated bond-angle distributions $\theta_{\mathrm{GeSeGe}}$ and $\theta_{\mathrm{SeGeSe}}$ for liquid $\mathrm{GeSe}_{2}$ at $T$ $=1050 \mathrm{~K}$. The results are given for the models NvdW (green line), vdW1 (red line) and vdW2 (blue line). These distributions have been calculated by including neighbors separated by less than $3 \AA$. 
Table I. Percentages of Ge atoms in edge-sharing, corner-sharing and homopolar bonding configurations

\begin{tabular}{lccc}
\hline \hline & $N_{\mathrm{Ge}}(\mathrm{ES})$ & $N_{\mathrm{Ge}}(\mathrm{CS})$ & $N_{\mathrm{Ge}-\mathrm{Ge}}$ \\
\hline$l-\mathrm{GeSe}_{2}(\mathrm{NvdW})$ & 45 & 36 & 18 \\
$l-\mathrm{GeSe}_{2}(\mathrm{vdW} 1)$ & 39 & 43 & 18 \\
$l-\mathrm{GeSe}_{2}(\mathrm{vdW} 2)$ & 41 & 36 & 23 \\
\hline \hline
\end{tabular}

more numerous in the vdW1 case, while the vdW2 scheme has little effect on the relative proportion of these quantities when compared to NvdW results. To quantify these observations we have calculated the number of Ge atoms belonging to corner-sharing and edge-sharing connections, $N_{\mathrm{Ge}}(\mathrm{CS})$ and $N_{\mathrm{Ge}}(\mathrm{ES})$ respectively, together with the number of Ge atoms found in homopolar bonds $N_{\mathrm{Ge}-\mathrm{Ge}}$. To this end we adopted the proposal of Ref. 58 , i.e. $N_{\mathrm{Ge}}(\mathrm{CS})=1-N_{\mathrm{Ge}}(\mathrm{ES})-$ $N_{\mathrm{Ge}-\mathrm{Ge}}$, which holds in the absence of extended chains. ${ }^{58}$

By using the values of $N_{\mathrm{Ge}}(\mathrm{CS})$ and $N_{\mathrm{Ge}}(\mathrm{ES})$ for the three models $\mathrm{NvdW}$, vdW1, vdW2 (see Tab. I) one obtains $R_{C S / E S}^{N v d W}=N_{\mathrm{Ge}}(\mathrm{CS}, \mathrm{NvdW}) / N_{\mathrm{Ge}}(\mathrm{ES}, \mathrm{NvdW})=0.8, R_{C S / E S}^{v d W 1}=N_{\mathrm{Ge}}(\mathrm{CS}, \mathrm{vdW} 1) / N_{\mathrm{Ge}}(\mathrm{ES}, \mathrm{vdW} 1)$ $=1.1$ and $R_{C S / E S}^{v d W 2}=N_{\mathrm{Ge}}(\mathrm{CS}, \mathrm{vdW} 2) / N_{\mathrm{Ge}}(\mathrm{ES}, \mathrm{vdW} 2)=0.87$. The experimental prediction of Ref. 24 points toward close percentages of edge-sharing and corner-sharing connections. Likewise, all FPMD models produced in the past are in line with values for $R_{C S / E S}$ moderately smaller than 1 , this meaning that the number of CS connection cannot be higher than the number of ES connections. Even though the values obtained for CS and ES in the vdW1 case are not too dissimilar when accounting for the statistical uncertainties, it remains true that a tendency to overestimate the CS/ES ration is noticeable. Therefore, the result corresponding to vdW1 for $R_{C S / E S}^{v d W 1}(1.1)$ is indicative of an artificial and yet, moderate contribution introduced in the FPMD model by the Grimme recipe. This contribution favors the CS arrangement and longer Ge-Ge distances for the inter-tetrahedral connections.

\section{CONCLUSION}

Liquid $\mathrm{GeSe}_{2}$ is a disordered network-forming material well suited to be exploited as benchmark system for model improvements and assessment of performances. In this case, we are interested in the impact of dispersion forces on the structural properties of this network, due to the existence of such effects in other disordered chalcogenide materials. Also, dispersion forces are 
a missing ingredient in the theoretical treatment of this prototypical liquid, for which numerous investigations by using different first-principles atomic-scale models have become available. To account for van der Waals dispersion forces, we have employed two schemes. In the first, due to Grimme, the coefficient expressing the intensity of the long range attractive forces does not evolve (i.e. is not updated) following the temporal evolution of the electronic structure. In the second, this requirement is met by resorting to the maximally localized Wannier functions. Overall, the set of structural properties considered here (partial structure factors and pair correlation functions, as well as standard fingerprints of network topology as the coordination numbers and the kind of the tetrahedral connections) are not significantly affected by the account of the dispersion forces. In particular, the maximally localized Wannier scheme leads to structural data very close to those obtained in the absence of dispersion forces. On the contrary, the Grimme approach introduces some spurious contributions clearly noticeable at the level of the partial pair correlation functions and the Ge-Se-Ge bond angle distribution. In terms of structural features, these correspond to a moderate overestimate of the number of corner-sharing connections at the expenses of the edgesharing connections. Our results provide convincing evidence on the superior reliability of the MLWF scheme in accounting for dispersion forces. It appears of paramount importance to rely on a methodology that does not modify structural properties when the dispersion forces have a negligible effect in comparison to other bonding effects. Further studies of this kind for disordered network systems at concentrations differing markedly from the stoichiometric one would be instrumental to confirm this conclusion.

\section{REFERENCES}

${ }^{1}$ K. Wezka, A. Bouzid, K. J. Pizzey, P. S. Salmon, A. Zeidler, S. Klotz, H. E. Fischer, C. L. Bull, M. G. Tucker, M. Boero, S. Le Roux, C. Tugène and C. Massobrio, Phys. Rev. B 90, 054206 (2014).

${ }^{2}$ A. Bouzid, K. J. Pizzey, A. Zeidler, G. Ori, M. Boero, C. Massobrio, S. Klotz, H. E. Fischer, C. L. Bull, P. S. Salmon, Phys. Rev. B 93, 014202 (2016).

${ }^{3}$ A. Bouzid, S. Le Roux, G. Ori, M. Boero and C. Massobrio, J. Chem. Phys. 143, 034504 (2015).

${ }^{4}$ A. Bouzid, S. Gabardi, C. Massobrio, M. Boero, and M. Bernasconi, Phys. Rev. B 91184201 (2015). 
${ }^{5}$ A. Bouzid, C. Massobrio, M. Boero, G. Ori, K. Sykina and E. Furet, Phys. Rev. B 92134208 (2015).

${ }^{6}$ A. Bouzid, H. Zaoui, P. L. Palla, G. Ori, M. Boero, C. Massobrio, F. Cleri and E. Lampin, Phys. Chem. Chem. Phys. 199729 (2017)

${ }^{7}$ S. Le Roux, A. Bouzid, M. Boero and C. Massobrio, Phys. Rev. B 86, 224201 (2012).

${ }^{8}$ S. Le Roux, A. Bouzid, M. Boero and C. Massobrio, J. Chem. Phys. 138, 174505 (2013).

${ }^{9}$ S. Caravati, M. Bernasconi, T. D. Kühne, M. Krack, and M. Parrinello, Appl. Phys. Lett. 91, 171906 (2007).

${ }^{10}$ S. Caravati, M. Bernasconi, T. D. Kühne, M. Krack, and M. Parrinello, J. Phys.: Condens. Matter 21255501 (2009); errata 21499803 (2009);

${ }^{11}$ S. Caravati, M. Bernasconi, and M. Parrinello, Phys. Rev. B 81, 014201 (2010).

${ }^{12}$ J. Akola and R. O. Jones, Phys. Rev. B 76, 235201 (2007).

${ }^{13}$ J. Kalikka, J. Akola, and R. O. Jones, Phys. Rev. B 90184109 (2014).

${ }^{14}$ J. Kalikka, J. Akola, and R. O. Jones, Phys. Rev. B 94134105 (2016).

${ }^{15}$ J. Hegedüs and S. R. Elliott, Nat. Mater. 7, 399 (2008).

${ }^{16}$ J. Y. Raty, W. Zhang, J. Luckas, C. Chen, R. Mazzarello, C. Bichara, and M. Wuttig, Nature communications 6, 7467 (2015).

${ }^{17}$ M. Micoulaut, J. Chem. Phys. 138, 061103, (2013).

${ }^{18}$ A. Bouzid, C. Massobrio, M. Boero, G. Ori, K. Sykina, and E. Furet, Phys. Rev. B 92, 134208 (2015).

${ }^{19}$ J. P. Perdew, K. Burke and M. Ernzerhof, Phys. Rev. Lett. 77, 3865 (1996); 78, 1396 (1997).

${ }^{20}$ A. D. Becke, Phys. Rev. A 38, 3098 (1988).

${ }^{21}$ C. Lee, W. Yang and R. G. Parr, Phys. Rev. B 37, 785 (1988).

${ }^{22}$ S. Grimme, J. Comput. Chem. 27, 1787 (2006).

${ }^{23}$ S. Grimme, J. Antony, S. Ehrlich, H. Krieg, J. Chem. Pys. 132, 154104 (2010).

${ }^{24}$ I. T. Penfold, P. S. Salmon, Phys. Rev. Lett. 67, 97 (1991).

${ }^{25}$ I. Petri, P. S. Salmon, and H. E. Fischer, Phys. Rev. Lett. 84, 2413 (2000).

${ }^{26}$ H. E. Fischer, A. C. Barnes, P. S. Salmon, Rep. Progr. Phys. 69, 233 (2006).

${ }^{27}$ C. Massobrio, A. Pasquarello, R. Car, Phys. Rev. B 64, 144205 (2001).

${ }^{28}$ C. Massobrio, A. Pasquarello, Phys. Rev. B 77, 144207 (2008).

${ }^{29}$ P. Biswas, D. N. Tafen, and D. A. Drabold, Phys. Rev. B 71, 054204 (2005).

${ }^{30}$ D. N. Tafen and D. A. Drabold, Phys. Rev. B 71, 054206 (2005). 
${ }^{31}$ J. C. Mauro and A. K. Varshneya, J. Am. Ceram. Soc. 89, 2323 (2006).

${ }^{32}$ C. Massobrio, A. Pasquarello, R. Car, J. Am. Chem. Soc. 121, 2943 (1999).

${ }^{33}$ M. Micoulaut, R. Vuilleumier, C. Massobrio, Phys. Rev. B 79, 214205 (2009).

${ }^{34}$ J. P. Perdew and Y. Wang, Phys. Rev. B 45, 13244 (1992).

${ }^{35}$ C. Massobrio, P. S. Salmon, M. Micoulaut, Solid St. Sciences 12, 199 (2010).

${ }^{36}$ A. B. Bhatia, D. E. Thornton, Phys. Rev. B 2, 3004 (1970).

${ }^{37}$ M. Micoulaut, S. Le Roux and C. Massobrio, J. Chem. Phys. 136, 224504 (2012).

${ }^{38}$ C. Yildirim, J.-Y. Raty, M. Micoulaut, J. Chem. Phys. 144, 224503 (2016).

${ }^{39}$ P. L. Silvestrelli, J. Phys. Chem. A 113, 5224 (2009).

${ }^{40}$ A. Ambrosetti, P. L. Silvestrelli, Phys. Rev. B 85, 073101 (2012).

${ }^{41}$ T. Ikeda, M. Boero, J. Chem. Phys. 143, 194510 (2015).

${ }^{42}$ J. Ruska, H. Thurn, J. Non-Cryst. Solids 22277 (1976).

${ }^{43}$ R. Car, M. Parrinello, Phys. Rev. Lett. 55, 2471 (1985).

${ }^{44}$ See http://www.cpmd.org, copyright 2000-2017 jointly by IBM Corporation and by Max Planck Institute, Stuttgart.

${ }^{45}$ N. Trouillier, J. L. Martins, Phys. Rev. B 43, 1993 (1991).

${ }^{46}$ F. Shimojo, Z. Wu, A. Nakano, R. K. Kalia, and P. Vashishta, J. Chem. Phys. 132, 094106 (2010).

${ }^{47}$ K. Koizumi, M. Boero, Y. Shigeta, and A. Oshiyama, J. Phys. Chem. Lett. 4, 1592 (2013).

${ }^{48}$ G. Ori, C. Massobrio, A. Bouzid, M. Boero, and B. Coasne, Phys. Rev. B 90, 045423 (2014).

${ }^{49}$ P. Hohenberg, W. Kohn, Phys. Rev. 136, B864 (1964).

${ }^{50}$ W. Kohn, L. J. Sham, Phys. Rev. 140, A1133 (1965).

${ }^{51}$ S. Grimme, A. Hansen, J. G. Brandenburg, C. Bannwarth, Chem. Rev. 116, 5154 (2016)

${ }^{52}$ P. Partovi-Azar, M. Berg, S. Sanna, T. D. Kuhne, Int. J. Quantum Chem. 116, 1160 (2016)

${ }^{53}$ S. Nosé, Mol. Phys. 52, 255 (1984).

${ }^{54}$ W.G. Hoover, Phys. Rev. A 31, 1695 (1985).

${ }^{55}$ P. E. Blöchl, M. Parrinello, Phys. Rev. B 45, 9413 (1992).

${ }^{56}$ A. Bhatia and D. Thornton, Phys. Rev. B 2, 3004 (1970).

${ }^{57}$ P. S. Salmon, J. Non-Cryst. Solids 353, 2959 (2007).

${ }^{58}$ I. Petri and P. S. Salmon, J. Phys. Condens. Matter 15, S1509 (2003). 